\title{
FOTOGRAFIE, AURA, ÜBERTRAGUNG: VON WALTER BENJAMIN ZU THOMAS STRUTH, GREGORY CREWDSON UND CARLOS GOLDGRUB ${ }^{1}$
}

\author{
Ulrich Johannes Beil ${ }^{*}$ \\ Universität Zürich
}

\begin{abstract}
Zusammenfassung: In diesem Aufsatz handelt es sich um den Versuch, drei international anerkannte Fotografen - Carlos Goldgrub aus Brasilien, Gregory Crewdson aus den USA und Thomas Struth aus Deutschland -, die in den letzten beiden Jahrzehnten hervorgetreten sind, am Beispiel ausgewählter Arbeiten mit Benjamins Essay Kleine Geschichte der Photographie in Beziehung zu setzen und zu zeigen, dass sie die dort erörterte 'Aura' auf je eigene Weise in den Blick nehmen und kreativ thematisieren. Dabei wird angenommen, diese Fotografen hätten Benjamins Text gelesen und sie hätten insbesondere dessen Ausführungen über die Aura jeweils in ihre Werke zu übertragen versucht.
\end{abstract}

Schlüsselwörter: Zeitgenössische Fotografie. Aura. Walter Benjamin. Carlos Goldgrub. Thomas Struth. Gregory Crewdson.

\footnotetext{
* Formação em Germanística, Filosofia, Teologia e Ciência Política, Universidade de Munique. Mestrado e Doutorado em Filosofia, Universidade de Munique. Tese de habilitação em Germanística e Literatura Comparada, Universidade de Munique. Professor assistente da Universidade de Hokkaido (1988-1992). Professor visitante da Universidade de São Paulo (2000-2004). Professor da Universidade de Munique e pesquisador sênior do grupo de pesquisa NFS Mediality, Universidade de Zurique, Suíça. E-mail: ubeil@hotmail.com
} 


\title{
PHOTOGRAPHY, AURA, TRANSMISSION: FROM WALTER BENJAMIN TO THOMAS STRUTH, GREGORY CREWDSON AND CARLOS GOLDGRUB
}

\begin{abstract}
The following article enters into the question if and how three well-respected contemporary photographers - Carlos Goldgrub from Brasil, Gregory Crewdson from the USA and Thomas Struth from Germany - refer to the works of Walter Benjamin. The article tries to show that the Benjaminian idea of „aura“, especially in the essay A short History of Photography, can be found in some works of the photographers and that they reflect and thematize this concept in different creative ways. The suggestion is: These photographers tried to transfer and to realize the conception of „aura“ in some examples of their work.

Keywords: Contemporary photography. Aura. Walter Benjamin. Carlos Goldgrub. Thomas Struth. Gregory Crewdson.
\end{abstract}

Schon wenn man bei Walter Benjamin nach einer Erläuterung des Aura-Begriffs sucht, stößt man auf etwas, das man Übertragung nennen könnte. Auf die Frage „Was ist eigentlich Aura?“ antwortet er in seinem Essay Kleine Geschichte der Photographie mit einem Satz, in dem Begriffe wie „Raum“ und „Zeit“ zwar auftauchen, aber sogleich mit einem „sonderbare[n] Gespinst“, einer „einmalige[n] Erscheinung“ in Verbindung gebracht werden - Phänomenen also, die einem nicht-begrifflichen Bereich entstammen. Noch deutlicher wird die Übertragung eines begrifflich-essayistischen in ein literarisch-poetisches Register in dem darauf folgenden Satz, in dem ein „Sommermittag“, ein „Gebirgszug“ und ein „Zweig“ die zentrale Rolle spielen, in dem also der Begriff der Aura in eine imago, einen bildlichen Zusammenhang übersetzt wird. Aber führen wir uns die berühmte Stelle als ganze vor Augen: 
„Was ist eigentlich Aura? Ein sonderbares Gespinst von Raum und Zeit: einmalige Erscheinung einer Ferne, so nah sie sein mag. An einem Sommermittag ruhend einem Gebirgszug am Horizont oder einem Zweig folgen, der seinen Schatten auf den Betrachter wirft, bis der Augenblick oder die Stunde Teil an ihrer Erscheinung hat - das heißt die Aura dieser Berge, dieses Zweiges atmen. “ 2

Diese Passage, die mit ihrem ereignishaften, apersonalen Duktus - „einem Zweig folgen“ - an ein heideggerisch gedachtes 'Sein' oder 'Anwesen' erinnert, kann man als das Kernstück, als die 'Urszene' der Benjaminschen Medienreflexion bezeichnen. Und dies, obwohl hier die Nähe zu medialen Verhältnissen so wenig gegeben zu sein scheint wie kaum sonst in Benjamins Werk. Man hat versucht, die Stelle intertextuell einzubinden und das vermeintlich 'Einmalige' ihres Auftretens zu relativieren: So hat man Anspielungen auf Baudelaires Prosagedicht Perte d'auréole, Prousts Recherche, Valerys Avant-propos oder einen Aphorismus von Karl Kraus heraushören wollen („Je näher man ein Wort ansieht, desto ferner schaut es zurück“), ${ }^{3}$ auch Alois Riegls „Stimmung“, Goethes Urphänomen oder das Kantsche Erhabene wurden angeführt. ${ }^{4}$

Entgegen seiner Verheißung bietet diese Passage aus der Kleinen Geschichte der Photographie, die später, geringfügig verändert, im Aufsatz über Das Kunstwerk im Zeitalter der technischen Reproduzierbarkeit wiederkehren wird, ${ }^{5}$ keine Begriffserklärung und keine Aufhellung des kulturellen Hintergrunds. Zwar berührt sich das hier vorgestellte Verständnis mit dem schon in der griechisch-lateinischen Antike geläufigen: aúra/aura als Lüftchen, Brise oder Glanz. Auch mag eine zeitgenössische, zwischen Wissenschaft und Esoterik oszillierende Begriffsverwendung im Hintergrund stehen: Aura als divina aura des paradiesischen Menschen in der Religionsphilosophie Franz von Baaders; als Vorbotin des epileptischen Anfalls in der Psychiatrie Charcots; als vom Körper des Menschen ausstrahlende Spur seines existentiellen 
Unterwegsseins in der lurianischen Kabbala, die Gershom Scholem rekonstruierte. ${ }^{6}$ Aber nichts von all dem wird explizit. Vielmehr gewinnt der Begriff sein Schillern gerade dadurch, dass er eine Fülle von Konnotationen in sich aufnimmt, sich gleichzeitig aus den geläufigen Kontexten herauslöst und auf einen neuen Zusammenhang bezieht: die Entwicklung von Medien. Ihnen gegenüber scheint die 'Aura' eine Art von 'Jenseits' zu vertreten, ein 'Anderes', umso mehr ersehnt, als sich der erfüllte Augenblick in der medialen Beschleunigung der Moderne zu zersetzen und verflüchtigen droht. ${ }^{7}$

Neben die Beschwörung der Aura als des EpiphanischUnverfügbaren tritt bei genauerem Hinsehen allerdings auch ein analytisches Moment. Differenzen werden erkennbar: zunächst die von Raum und Zeit, den apriorischen Anschauungsformen Kants; dann die von Absenz und Präsenz, die durch Ferne und Nähe, den „Gebirgszug" und den „Zweig“ konkretisiert werden; schließlich die zwischen den genannten 'Gegenständen' der Natur und dem, der sie betrachtet, Objekt und Subjekt. Es zeigt sich: Das, was hier in lyrisch anmutenden Worten zu einer atmosphärischen Einheit zu verschmelzen scheint, basiert auf kategorialen Unterschieden, die erst nachträglich, im Rahmen einer poetischen (und nicht argumentativen) Syntax, miteinander vermittelt werden.

Das „Gespinst“, das die Kategorien von Raum und Zeit verknüpft, die „Erscheinung“, in der Ferne und Nähe sich übereinander blenden, die Wahrnehmung, in der sich optische, haptische und olfaktorische Momente zu einem einzigen Eindruck verbinden, beruhen auf einem sekundären, einem synthetischen Akt. Dieser Akt bringt Bildliches nicht unmittelbar, als Selbstevidenz, zur Erscheinung, sondern mittelbar, als Übertragung begrifflicher Konstellationen. Statt als extramediales Jenseits erweist sich 'Aura' als immer schon in Wahrnehmungsbedingungen involviert - als Teil jener Relationen, die sie zu transzendieren scheint. Von daher sind Momente der Vermittlung, der Reproduktion und der Übertragung weniger Antipoden der Aura oder Ausdruck ihrer Verfallsgeschichte. Vielmehr sind sie in ihr Konzept, wie es eine 
genaue Lektüre von Benjamins Text erkennen lässt, von Anfang an als Bedingung ihrer Möglichkeit eingeschrieben - ebenso wie die Momente der Perspektivierung und der historischen Differenz. ${ }^{8}$

Bevor nun Benjamins Essay mit Beispielen gegenwärtiger Kunstfotografie in Beziehung gesetzt wird, zunächst noch einige Bemerkungen zur Kleinen Geschichte der Photographie. Der Text, den Benjamin 1931 schreibt, ist zunächst als Rezension verschiedener neuerer Photographiebände gedacht, weitet sich aber zu einer grundsätzlichen Reflexion. ${ }^{9}$ Behandelt werden zunächst, nach einem kurzen Vergleich der Anfänge der Photographie mit denen des Buchdrucks, die Bedingungen der Möglichkeit, sich dem relativ jungen Medium historisch anzunähern. Diese seien gerade heute, in einer Phase der „Erschütterung der kapitalistischen Industrie“, gegeben. Die Krise erlaube es, sich der „Blüte der Photographie“, die in das erste vorindustrielle Jahrzehnt, also die 1840 er Jahre, falle, bewusst zu werden (368). Die sich andeutende Abgeschlossenheit einer Epoche biete die Möglichkeit, auf diese zurückzublicken - in einer „kleinen“ Geschichte, die das, was ihr zur größeren fehlt, durch geschichtsphilosophische Perspektiven wettmacht, die sich auf die Eigenlogiken der photographischen Entwicklung richten. Benjamin verfährt einerseits immanent, nach dem Schema von Aufstieg, Verfall und gegenwärtiger Konsolidierung, wie sich dies ähnlich auch bei anderen Historikern des Genres - Franz Roh, Heinrich Schwarz, Camille Recht oder Emil Orlik - findet. ${ }^{10}$ Er geht aus von den Erfindern und frühen Virtuosen der Lichtbildkunst wie Daguerre, Nièpce, Nadar und Hill, die die ursprüngliche Dignität und Einzigartigkeit des Genres repräsentieren. Er verfolgt die weitere Entwicklung anhand des Wandels der reproduktiven Techniken und der Ausbreitung der Popularphotographie, deren überladene Studiokulissen die These vom Verfall besonders einleuchtend illustrieren. Und er endet mit Photosammlungen Atgets, Sanders, Bloßfeldts, Krulls, die zwar die Aura preisgegeben haben, aber doch für neue, ungeahnte Wahrnehmungsmöglichkeiten sorgen, indem sie mit ihren beweglicheren Kameras »flüchtige und geheime 
Bilder« festzuhalten vermögen (385). Andererseits nimmt er eine komparatistische Perspektive ein. Er positioniert die Photographie in der gesamtkulturellen Entwicklung ihrer Zeit, beobachtet von ihr ausgelöste Modifikationen des Kunstbegriffs und betrachtet sie in ihrer Beziehung zu anderen Künsten, insbesondere zur Malerei. Nicht nur im Bereich der Landschaftsmalerei sei die Photographie es, welche die „Strafette" übernommen habe, auch und gerade die Porträtminiatur werde zum „Opfer" der neuen technischen Errungenschaft; im $\mathrm{Nu}$ seien aus Miniaturmalern Berufsphotographen geworden (374). Zur Debatte steht für Benjamin nicht nur, ob die Photographie selbst eine „Kunst“ sei, sondern auch, inwiefern sie die „Funktion“ jener Kunstwerke, zu deren Reproduktion sie tagtäglich beitrage, verändert habe (381). Immer von neuem umkreist er, auch mit Hilfe entsprechender Äußerungen von László Moholoy-Nagy oder Tristan Tzara, die Spannungen, die sich zwischen den traditionellen und den aktuellen Bildkünsten ergeben, erörtert er Momente der Antizipation, Konkurrenz und wechselseitigen Beeinflussung, des Ökonomischen und des Modischen.

Soweit einige Stichworte zu Struktur und Ausrichtung des Benjaminschen Textes. Aura wird dort, wie sich zeigte, der reproduktiven Technik der Fotografie, ähnlich wie später im Kunstwerk-Aufsatz, keinesfalls rundweg abgesprochen. Vielmehr wird sie dem Medium in seiner frühen "Blütezeit" sehr wohl zugestanden, jedenfalls solange der Prozess der „Verdrängung des Dunkels durch lichtstärkere Objektive" ebenso wenig eingesetzt hatte wie der Wirklichkeitsverlust des „imperialistischen Bürgertums“ (377). Von jenem nostalgischen „Hauchkreis“ ist in diesem Zusammenhang die Rede, von der ,altmodische[n] ovale[n] Form des Bildausschnitts“, in denen "Objekt und Technik“ sich noch genau entsprachen (376). Da sich aber, Benjamin zufolge, der "Verfall“ der Aura schon nach dem ersten Jahrzehnt der Fotografie beobachten lässt, neigte man vielfach dazu, anzunehmen, dass die Aura - so sehr man sie auch später durch „alle Künste der Retusche“ (377) wiederherzustellen versuchte - seither weitgehend 
vergebliches Desiderat geblieben ist. Liest man Benjamins Text derart monolinear und ignoriert all jene Stellen, die den Zusammenhang Technik/Aura, Medialität/Aura favorisieren, so bleiben nur zwei Optionen: erstens, die vielfach kolportierte These vom Verfall der Aura, allen Widerständen in Benjamins Text zum Trotz, aufrecht zu erhalten. Das hieße nichts Anderes, als dass den Künsten seit Mitte des 19. Jahrhunderts ihre auratische Dimension ein für alle Mal verloren gegangen ist. Die zweite, weit häufiger gewählte Option: Man müsse die ganze Fragestellung als obskur und unproduktiv verwerfen - frei nach Brecht, der Benjamins Aura-Reflexionen bekanntlich "ziemlich grauenhaft“ fand. ${ }^{11}$

Im Widerspruch zu diesen beiden Optionen sei im Folgenden ein kleines Experiment durchgeführt. Es handelt sich um den Versuch, drei international anerkannte Fotografen - Carlos Goldgrub aus Brasilien, Gregory Crewdson aus den USA und Thomas Struth aus Deutschland -, die in den letzten beiden Jahrzehnten hervorgetreten sind, am Beispiel ausgewählter Arbeiten mit Benjamins Text in Beziehung zu setzen. Dabei geht es nicht um eine Veranschaulichung von dort vertretenen Thesen, sondern um die fiktive Unterstellung, diese Fotografen hätten Benjamins Text gelesen - was bei der akademischen Ausbildung der Drei ohnehin anzunehmen ist - und sie hätten insbesondere dessen Ausführungen über die Aura auf je andere Weise in ihre Werke zu übertragen versucht. Übertragen heißt hier, um es noch einmal zu betonen, nicht illustrieren. Vielmehr geht es darum, in der anderen, nicht-literalen Kunst der Fotografie, die bei Benjamin thematisiert wird, auf zentrale Annahmen des Essays zu reagieren, sie zu dekonstruieren, zu widerlegen oder auf verquere Weise zu bestätigen. Dass bei diesen Übertragungen die spezifisch medialen Bedingungen der Fotografie eine wichtige Rolle spielen, sei gleich vorweg betont, ebenso die Beobachtung, dass alle drei Fotografen inter- bzw. transmedial verfahren, um ihrer 'Arbeit an der Aura' Nachdruck zu verleihen. 
Von den hier zu behandelnden Fotografen ist Thomas Struth derjenige, der der Benjaminschen Aura-Konzeption prima vista am nächsten zu kommen und sie auf seine Weise und mit seinen Mitteln geradezu bestätigen zu wollen scheint. Der 1954 in Geldern am Niederrhein geborene Künstler, der zunächst bei Gerhard Richter und Peter Kleeman Malerei studierte und der Düsseldorfer Schule zugerechnet wird, gehört zu den bekanntesten deutschen Fotografen. Auch wenn die Spannbreite seiner Sujets beträchtlich ist - es finden sich Natur- und Stadtaufnahmen ebenso wie Familienporträts -, so scheinen doch die Museum Photographs für seine metareflexive und intermedial verfahrende Arbeit besonders charakteristisch zu sein. Nach einigen Vorstudien, etwa im Louvre und im Musée d'Orsay, begann er ab 2001, etwa im Berliner Pergamon-Museum, diese Fotografien auch zu inszenieren. Im Mittelpunkt dieser Arbeiten stehen in der Regel klassische Gemälde oder Skulpturen, die nicht für sich, sondern in ihrem gegenwärtigen Ausstellungskontext gezeigt werden, also im Museumsraum mit den jeweils wie zufällig davor stehenden Betrachtern. Nach einer Äußerung des Künstlers ging es zunächst weniger um ein auf Aura spekulierendes, als vielmehr um ein rezeptionstheoretisches, ja demokratisches Anliegen: Die Idee hinter den „Museumsfotografien“ sei es gewesen, „Meisterwerke dem Schicksal des Ruhmes zu entreißen, sie von ihrem Status als ikonische Gemälde zu befreien“ und an jenen „gegenwärtigen Moment“ zu erinnern, in dem sie geschaffen wurden. „Kurz gesagt: es ging mir darum, die Zeit des Bildes und die Zeit des Betrachters miteinander zu verknüpfen “. ${ }^{12}$ Auch wenn diese Selbstaussage geradezu anti-auratisch klingt - mit Fotografien wie denen vom Dürer-Selbstbildnis aus der Alten Pinakothek in München oder Jan Vermeers Lautenspielerin am Fenster aus der National Gallery in London mit ihren teilweise oder ganz ausgeblendeten Betrachtern schlägt Struth offensichtlich einen anderen Weg ein.

Sehen wir uns die zuletzt genannte Arbeit etwas genauer an (Abb. 1). ${ }^{13}$ In dem Bild gibt es auf den ersten Blick keinen 
Betrachter: Vermeers Gemälde ${ }^{14}$ (Abb. 2) hängt in einem graublau getönten Museumsraum. Struth hat es in die Nähe der rechten oberen Ecke des Fotos gerückt; man sieht es schräg von der Seite, es nimmt nicht einmal ein Viertel der Gesamtfläche ein. Der Raum außerhalb des Gemäldes bleibt leer. Was zunächst ins Auge fällt, ist die Staffelung von Rahmen. Diese beginnt schon in Vermeers Gemälde selbst, in dem - ebenfalls im rechten obere Eck - eine Europakarte zu sehen ist, die einen inneren und einen äußeren Rahmen aufweist. In ihrer Betonung des rechten Winkels und des linken unteren Ecks wiederholt sie jenen anderen rechten Winkel, den der Tisch der Lautenspielerin zusammen mit dem Fenster bildet. Diese Rahmungen setzen sich in dem komplexen, mehrfach gerillten Rahmen des Museumsbildes fort, in dessen matt schimmernden Holzleisten und in seinem Schatten, sie setzen sich weiterhin fort auf der Fotografie, wenn Struth die Ecke des Museumsraums mit ihrem rechten Winkel langsam aus dem Dunkel treten lässt - eine Struktur, an der schließlich auch noch die Kordel Anteil hat, die über den Boden gespannt ist und die Besucher auf Distanz hält (sie spielt auch auf die untere Leiste der Europakarte an, die wiederum auf das Ohr der Lautenspielerin zielt). Damit sich diese Mis-en-abyme-Struktur so wirkungsvoll entfalten kann, bedarf es eines (unsichtbaren) Betrachters, der nicht frontal auf das Gemälde starrt, sondern, in einer Art kreativer Zerstreutheit, das, was die museale Umgebung an subtilen Analogien bietet, wahrzunehmen und in seine Betrachtung einzubeziehen vermag. Hier lässt sich bereits an einen Benjaminschen Begriff denken, der die von spezifisch fotografischen Techniken erzeugten ungewöhnlichen Wahrnehmungen bezeichnet, nämlich den des „Optisch-Unbewussten“ (371). 


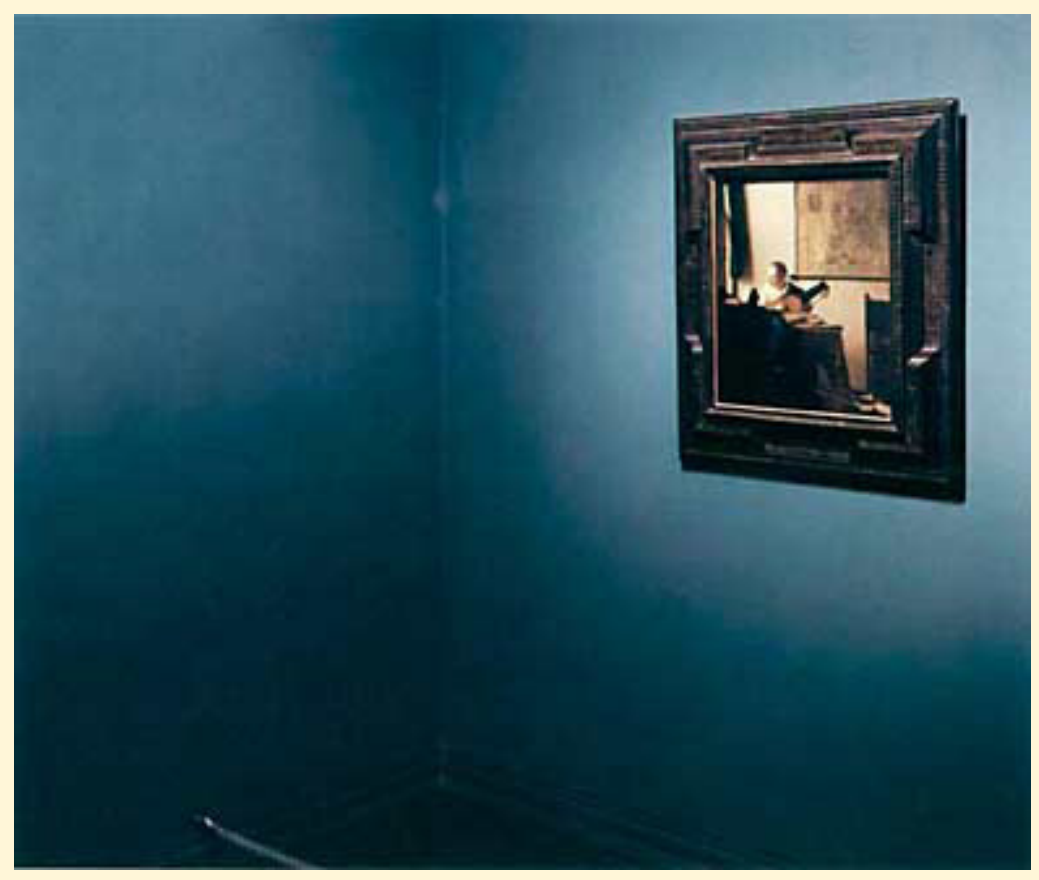

Abb. 1: Thomas Struth, National Gallery 2, London 2001 (aus: Museum Photographs, 2005) 


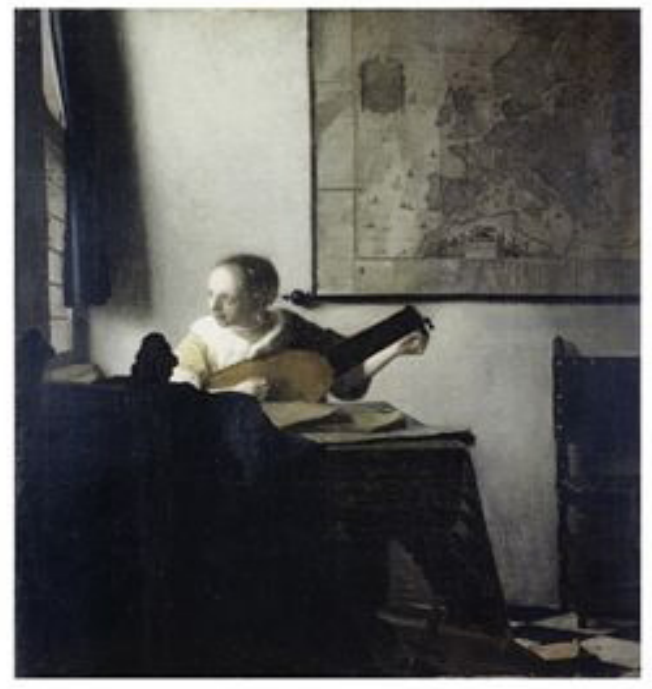

Abb. 2: Jan Vermeer, Lautenspielerin am Fenster (1662/68), Metropolitan Museum of Art NY

Aber dies ist nicht das einzige Moment, das an Benjamin erinnert. Ein anderes, augenfälligeres bietet die komplexe Lichtinszenierung im Raum. Keine Lichtquelle ist zu sehen, weder eine künstliche noch eine natürliche. Vielmehr scheint Vermeers Bild derart aus sich selbst heraus zu leuchten, dass dieser Lichtschein sowohl das Gemälde umgibt, als auch auf der linken Wandseite als kaum merklicher Schimmer, als 'Gepenst' des Gemäldes, sich wiederholt. Mit diesem Lichtschein gelingt es Struth, eine 'mystische' Stimmung hervorzurufen - zu der sich auch Benjaminsche Begriffe aus der Kleinen Geschichte wie „Schweigen“, „Abgeschiedenheit“, „Sammlung“, 'Dauer' assoziieren lassen (373). Darüber hinaus trägt die im Kunstwerk-Aufsatz gemachte Unterscheidung von Kultwert"/ "Ausstellungswert" möglicherweise zur Klärung dessen, 
was hier vorgeführt wird, bei. Diese Unterscheidung, an der sich dort das Vorhandensein bzw. der Verfall der Aura ablesen lässt, wird von Struth nun offensiv unterlaufen. Wenn es bei Benjamin heißt, „[i]n der Photographie“ beginne der „Ausstellungswert den Kultwert auf der ganzen Linie zurückzudrängen“, ${ }^{15}$ so kehrt Struth diese Aussage ins Gegenteil - als wolle er sagen: Die Fotografie sieht sich nicht nur in der Lage, dem Ausstellungswert (eines Gemäldes) kultische Elemente abzutrotzen, sondern auch und gerade dazu, den Ausstellungswert selbst in den Kultwert zurückzuverwandeln. Hierzu trägt nicht zuletzt auch das synästhetische, Vermeers Bild von der Lautenspielerin in eine Art Hallraum versetzende Moment bei. Die Fotografie legitimiert sich so als eine der Malerei ebenbürtige Kunst, da sie ihr zu ihrer Aura verhilft, ihre Aura definiert und so zur 'Wiederauferstehung' der Aura in den Kontexten der Gegenwart beiträgt. An der Tendenz zur Auratisierung kann nach all dem kaum ein Zweifel bestehen: Die Staffelung von Rahmungen, die einen mise-en-abyme-artigen Unendlichkeitseffekt erzeugen, tragen zur auratisierenden Wirkung dieser intermedialen Inszenierung - Gemälde/Holzschnitzerei/ Architektur - ebenso bei wie Benjamins "Hauchkreis“, das „absolute[] Kontinuum von hellstem Licht zu dunkelstem Schatten“ (376). Dieses „Kontinuum“ überträgt Struth von der frühen Fotografie, an der Benjamin es beobachtet, auf die Malerei, um es, so vermittelt, wieder in die Fotografie zurückkehren zu lassen, zu der es gehört. Man gewinnt den Eindruck, als versuche Struth, im Medium der Fotografie einen Diskurs über Aura zu führen, so wie Benjamin ihn im Medium der Schrift führt - einem Medium, das Aura aus perspektivischer, historischer Distanz thematisiert und so unter ähnlichen Bedingungen arbeitet wie die Fotografie.

\section{III}

Wenn schon in Struths Fotografien das Ineinandergreifen unterschiedlicher Medien eine wichtige Rolle spielt, so gehört 
unter den zeitgenössischen Fotografen gewiss Gregory Crewdson $\mathrm{zu}$ denjenigen, die diese inter- oder transmediale Tendenz zum Äußersten treiben. Die Arbeiten des 1962 in Brooklyn geborenen Künstlers mögen in einigen Aspekten an Jeff Wall (die aufwändige Inszenierung der Fotos), an Diana Arbus (das Abseitige, Unheimliche) oder Cindy Sherman (Untitled Film Stills) erinnern; vor allem hat man sehr früh die Bezüge zum Kino, zu Alfred Hitchcock, Steven Spielberg und vor allem David Lynch beobachtet, insbesondere zu dem Film Blue Velvet. ${ }^{16}$ Dennoch sind seine Fotografien längst unverwechselbar. Mit seinen unheimlichen, verwaisten oder schockierenden Szenerien, die wie für einen Film gemacht erscheinen und mit Hollywood-artigen Aufwand hergestellt sind, greift Crewdson große amerikanische Mythen auf. Die Eroberung der Welt durch das Automobil zum Beispiel - ein Traum, den er in seinen Straßenbildern immer wieder zum Alptraum werden oder einfach an einer Kreuzung scheitern lässt. ${ }^{17}$ Erst neuerdings beginnt man, Crewdsons Arbeiten auch mit Malerei in Verbindung zu bringen: Dies nicht nur, weil man bei ihm immer wieder modernen Klassikern wie Edward Hopper zu begegnen meint, sondern auch im Hinblick auf manieristische, barocke oder spätmittelalterlich-rinascimentale ikonographische Traditionen. Traditionen, von deren überwiegend geschlossenen, motivisch streng verfugten Bildordnungen Crewdson zweifellos gelernt hat. An vormoderne Kunstwerke fühlte man sich durch Crewdsons Clair-obscur, durch seine „Schaffung eines 'geheimnisvollen' Lichts “ erinnert $^{18}$ - durch eine Lichtregie, in der die „christliche Symbolik mit Szenen der Apotheose, Verklärung, Epiphanie und der Verkündigung an Maria“ im säkularen, (post-) modernen Kontext wiederzukehren scheint.

Als prägnantes Beispiel für diese Lichtregie kann Plate 51 aus dem Twilight-Zyklus gelten (1998-2002). ${ }^{19}$ Auf dieser Plate (Abb. 3) sieht man eine mit vereinzelten Holzhäusern gesäumte menschenleere Straße, die in einen Erdplatz im Vordergrund, auf dem Balken lagern, mündet. Es ist Abend. Der kaum von Wolken bedeckte Himmel durchquert ein Farbkontinuum von 
Dunkel-Türkis (oben) bis zu einem zarten Orangerosa über den Baumwipfeln (am Horizont). Was vor allem ins Auge fällt, ist ein kräftiger, von links oben nach rechts unten verlaufender Lichtstrahl, der das Bild wie eine Diagonale teilt. Am irrealen Charakter dieses Strahls lässt Crewdson keinen Zweifel: Er kann nicht von der Sonne ausgehen (die ist fast untergegangen), auch nicht vom Mond (dazu ist er zu stark gebündelt), aber eigentlich auch nicht von einer anderen natürlichen Lichtquelle (einem Leuchtturm hier, in dieser Vorstadt? Einem polizeilichen Suchscheinwerfer in zwanzig Meter Höhe?). Man kommt kaum umhin, an ein lumen supranaturale zu denken, ${ }^{20}$ das 'von oben', 'von außen' in diese menschenleere Szenerie einbricht. Aber worauf fokussiert dieser ominöse Strahl? Er zielt auf eine Stelle halb rechts, zwischen Straße und Wiese, einen Eck- und Endpunkt, von dem an sich nur noch gestampfte Erde in den Vordergrund erstreckt. Es ist jene Stelle im Bild, die besonders undefiniert wirkt - ein blinder Fleck in der ansonsten stillen, aufgeräumten Vorstadtordnung. Diese Szene, in der kaum mehr zu erkennen ist als zwei Schilder (von hinten), ein niedergerissener Maschendrahtzaun, Unkraut und einige am Boden liegende Gegenstände, Zaunpfähle etwa, gewinnt Bedeutsamkeit einzig durch den Lichtstrahl. Wie ein Spot scheint er diesen 'Verhau' aus seiner dunklen Umgebung hervorheben zu wollen. Warnen die Schilder mit ihrer unsichtbaren Schrift vor irgendeiner Gefahr? Zeigen sie ein Verbot an? Oder sollen sie nur mit ihrer Form - neben den Telegrafenmasten, den Balken und der christbaumartigen Tanne links - an das christliche Kreuz erinnern und damit an eine paradigmatische Durchdringung von Immanenz und Transzendenz? Verdient selbst dieses notorisch übersehene, winzige Niemandsland - eine Art Beckettsches Nazareth - einen Augenblick der Epiphanie? 


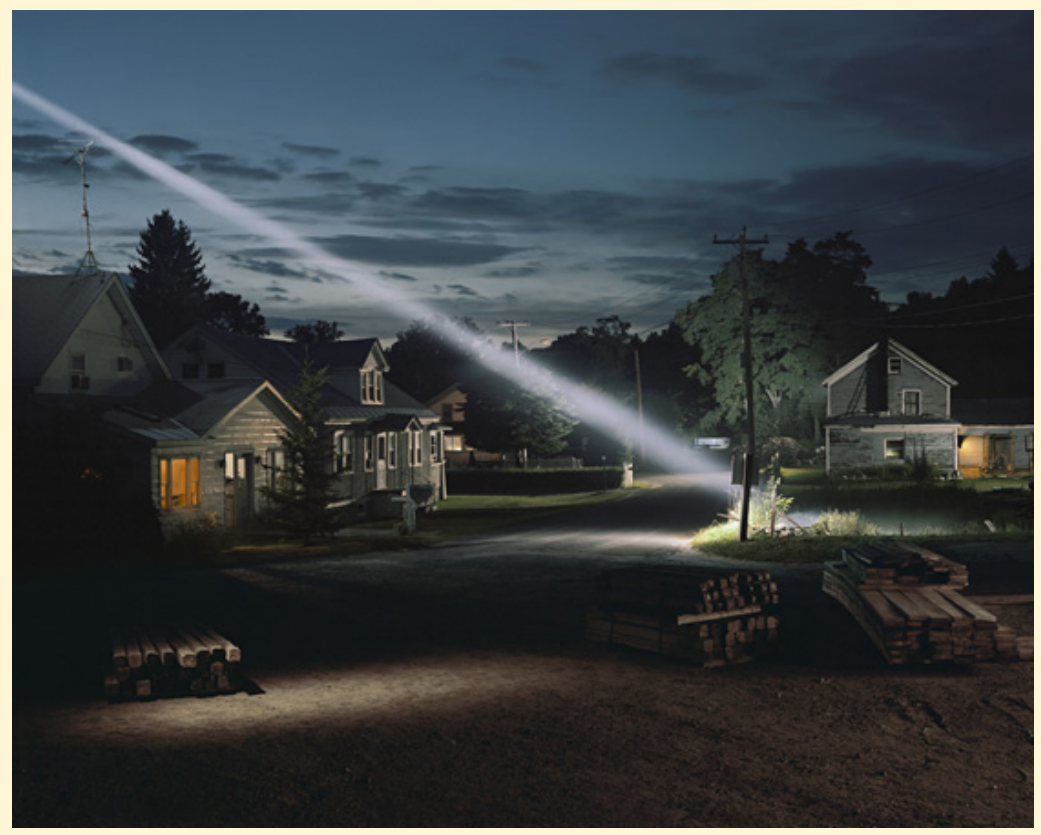

Abb. 3: Gregory Crewdson, Twilight, Plate 51 (2002)

Diese Spur weiterzuverfolgen, hieße, sich klassische Verkündigungsszenen zu vergegenwärtigen: die Verkündigungen des Pintoricchio (in Santa Maria Maggiore in Spello) etwa oder die des Philippe de Champaigne, in denen der Lichtstrahl von rechts oben nach links unten fällt. Enger mit Crewdsons Konzept dürfte die Annunciazione des Fra Angelico von 1430 zu verknüpfen sein, in der ein kräftiger goldener Lichstrahl diagonal von links oben nach rechts unten durch das Bild geführt wird (Abb. 4); an Plate 51 erinnern weiterhin die in abendlich verglimmendes Türkisblau getauchte Szenerie, die Pflanzen, das Holz (eine Bank im Hintergrund) sowie die Vertikalen - bei Fra Angelico Säulen, bei Crewdson Telegrafenmasten und Hausecken. ${ }^{21}$ Nicht, dass Crewdson religiöse Bildmotive in modernem Gewand wiederbeleben wollte, nein: Gerade dadurch, dass er sie zugleich zitiert und deformiert, aufruft 
und durchstreicht, kann er die einstige Aura als Form, von ihren Inhalten losgelöst, herauspräparieren, ironisch reinszenieren und eine von allen guten Geistern verlassene (Post-)Moderne an ihren Verlust erinnern. Wenn man auf den Gedanken kommen kann, Crewdson versuche in dieser und anderen Plates, auranahe Begriffe Benjamins wie „Schweigen“, „Abgeschiedenheit“, „Sammlung“ und 'Dauer' in seine Bildkompositionen zu übersetzen, so liegt dies durchaus auf dieser 'metaphysischen' Linie.

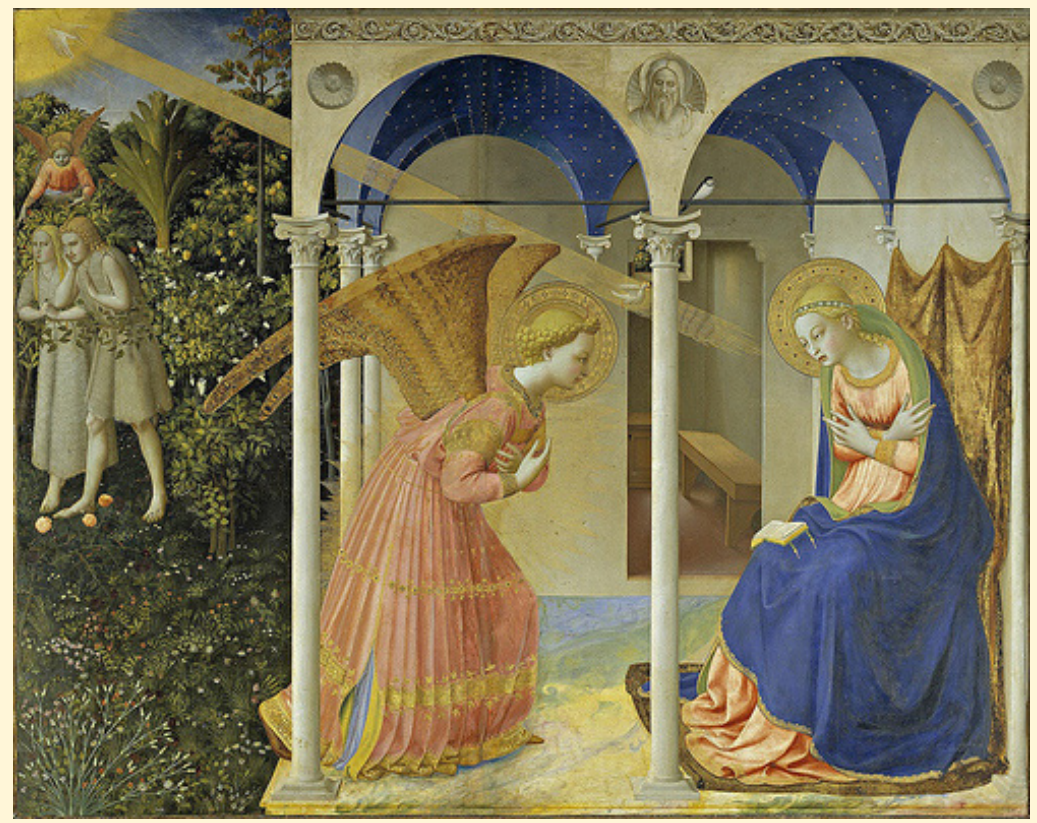

Abb. 4: Fra Angelico, Annunciazione (1430/32), Prado

Zugleich fühlt man sich, wie oft bei Crewdson, an jene 'Tatorte' erinnert, die Benjamin zum Markenzeichen der 'modernen' Fotografie Eugène Atgets erklärt hat. Sein Satz „Aber ist nicht jeder Fleck unserer Städte ein Tatort? Nicht jeder ihrer Passanten ein Täter?“ (385) könnte als Motto über eine Reihe 
von Arbeiten Crewdsons stehen - Arbeiten, die immer wieder die »Suggestionskraft« »unbekannter Tatort[e] « ausstrahlen. ${ }^{22}$ Die Differenz der Crewdsonschen 'Übertragung' besteht allerdings darin, dass die Tatort-Atmosphäre bei ihm die Aura nicht auslöscht, sondern sie im Gegenteil auf eigentümliche, mitunter bestechende Weise hervorbringt. Zum Gelingen dieses Effekts einer unheimlichen Aura, einer Aura, in der das Heilige und das Bedrohliche, das Epiphanische und das Alltägliche sich auf so undurchschaubare wie faszinierende Weise vermischen - man denke an die griechisch-lateinische Doppelbedeutung von hágios/ sacer, heilig und verflucht -, trägt die Subtilität des transmedialen Arrangements bei. Crewdson legt es, gerade auch in Plate 51, darauf an, Elemente der Malerei, der Architektur, des Theaters, der Literatur und natürlich des Films derart unauffällig ineinander zu blenden, dass sich die semantische Dichte seiner Inszenierung und damit ihrer Aura - potenziert.

Auratisiert wird so über den jeweils ins Licht gerückten alltäglichen Gegenstand und die verwendeten Medien hinaus auch und vor allem die Fotografie selbst. Sie, die zeitweise zwischen der 'eigentlichen' Kunst, der Malerei, und der massenmedial erfolgreichsten Kunst, dem Kino, aufgerieben zu werden drohte, empfiehlt sich hier als Kunst des Dazwischen - zwischen klassischer Malerei und modernem Film. Sie beansprucht eine vermittelnd-synthetische Position und agiert im Wortsinn transund multimedial. Es wird so der Eindruck erzeugt, als seien es die beiden entscheidenden Referenz-Medien, Malerei und Film, die in der Krise stecken und auf diese Weise 'gerettet', vor dem Vergessen bewahrt werden können.

\section{IV}

Als einziger der hier behandelten Fotografen widersteht Carlos Goldgrub der Versuchung, die Benjaminsche Aura mit spezifisch fotografischen Mitteln realisieren zu wollen. Goldgrub 
scheint die de-auratisierende Rolle, die Benjamin der modernen Fotografie als einer ausgesprochenen Reproduktionskunst zugeschrieben hat, nicht nur mehr oder weniger bereitwillig zu akzeptieren, sondern sie auch mit neuen, ungeahnten Energien zu erfüllen. Das Modell, dem er hier folgt, ist der von Benjamin im letzten Teil seines Photographie-Aufsatzes in den Blickpunkt gerückte französische Fotograf Eugène Atget, der auch schon bei den Crewdsonschen Tatorten Pate gestanden hat. Carlos Goldgrub, 1952 in Buenos Aires geboren, aber seit Mitte der 80er Jahre vorwiegend in Brasilien für verschiedene Zeitungen und Magazine tätig, machte durch eine Reihe von Ausstellungen, etwa auch zur jüdischen Immigration in Lateinamerika, auf sich aufmerksam. Er erhielt mehrere Auszeichnungen, unter Anderem den renommierten Grande Prêmio Ayrton Senna de jornalismo im Jahr 1999. Es ist insbesondere die 2003 unter dem Titel São Paulo - Um Retrato Urbano in der Sammlung Pirelli/MASP gezeigte Fotoserie Outdoors, die die ästhetische Eigenart und Radikalität erkennen lässt, zu der dieser Künstler fähig ist. ${ }^{23}$ Die durchweg in Schwarz-Weiß gehaltenen Arbeiten aus Outdoors sind es auch, die an Atget denken lassen. Sie erwecken den Eindruck, als versuche Goldgrub, Benjamins Charakterisierung des Pariser Fotografen in die Situation der Post- und Nachmoderne zu übersetzen und die Atget-Perspektive auf die multikulturelle, so faszinierende wie problembeladene Megacity São Paulo anzuwenden, wie sie sich dem Besucher nach der Jahrhundertwende, also in den Jahren 2000 bis 2003, zeigt. Auch wenn man immer wieder und nicht ohne Grund die Meinung geäußert hat, im São Paulo von heute sei keinerlei Flanieren mehr möglich, es herrsche das Diktat motorisierter Fortbewegung - schon 1922 nannte der Dichter Mario de Andrade einen Lyrikband Paulicéia desvairada (,Wahnsinnsstadt São Paulo'),$-^{24}$ so erscheinen Goldgrubs Outdoors doch als ein letzter verzweifelter Versuch, in einer der neuen 20-Millionen-Städte den Blick des Flaneurs für die Kunst zu retten. „São escolhas de um fotógrafo flâneur“ betont denn auch Dirce Carrion in ihrer Einführung zu der Serie. ${ }^{25}$ 
Aber wie kann es gelingen, jene von Benjamin hervorgehobene 'Leere' eben dort aufzuspüren, wo man prinzipiell auf alles Andere, nur nicht auf Leere stößt? „Merkwürdigerweise sind aber fast alle diese Bilder leer“, schreibt Benjamin über Atget (379). Goldgrub behilft sich mit einer Verschiebung der Perspektive: Statt sich auf die Straßen, ihre Fahrzeuge und vorüber hastenden Menschen $\mathrm{zu}$ konzentrieren und die horizontale Sehweise (des normalen Passanten) einzunehmen, richtet er seinen Blick vorwiegend nach oben, auf den Himmel über der Stadt. Was man sieht, sind Vertikalen - Skyscraper, Wohntürme, Antennen und Hochspannungsmasten, jene Nicht-Orte also, die dem gewöhnlichen Leben in der Regel entzogen sind. Insbesondere interessiert er sich für die haushohen Plakate, die eye-catcher der Konzerne, die in absurde Dimensionen vergrößerte Körper und Gesichter zu Werbezwecken zeigen und die oft unansehnlichen Fassaden vergessen lassen. Die Beschriftung, den Produktnamen allerdings spart er ebenso sorgsam aus wie das Gewimmel der Passanten an den Sockeln der metropolitanen édificios. Was Carlos Goldgrub zeigt, ist ein menschenleeres São Paulo, das, um mit Márcio Seligmann-Silva zu sprechen, „von riesigen Wesen dominiert wird, einer Art erhabener Halbgötter [...]. Diese Wesen, die das Schönheitsideal von den Titelseiten der Modezeitschriften verkörpern, leben in einer Idylle, während ringsherum Millionen von Menschen im Smog ersticken und in einer Explosion von Lärm und Gewalt versinken. [...] Sie posieren über der Stadt, als hätten sie Flügel, stellen ihre Schönheit ebenso zur Schau wie ihren 'Triumph' über den Tod und die Vergänglichkeit". ${ }^{26}$ Seligmann-Silva betont den "Sexappeal des Anorganischen“, den diese Wesen ausstrahlen, er vergleicht sie mit „Replikanten“, die „perfekt und doch zugleich von der Krankheit raschen Verfalls betroffen“" seien. Die von den Plakaten prangenden Figuren sind so zugleich medial und abstrakt. Sie sollen den Passanten kraft ihrer konsumistischen Transzendentalität verführen, anspringen, zum Kauf überreden. Sie, die wie Aliens aus einem planetarischen Utopia über dem Stadtgeschehen schweben, gleichen Verkörperungen den architektonischen Abstraktionen und 
Immunitäten. Nicht zuletzt erzeugen sie auf ihre Weise jenes surreale Moment, das Benjamin in den Fotografien Atgets wahrgenommen hat. Die politisch-kritische Funktion dieser Arbeiten lässt sich vor diesem Hintergrund mit Gewinn analysieren. Fasst man die Bilder allerdings (auch) als Übertragungen des Benjaminschen Atget-Modells auf, so gilt es darüber hinaus nach der Aura zu fragen, nach jenem atmosphärischen Moment, das die Fotografie im Verlauf ihrer - zur Zeit Benjamins noch kaum ein Jahrhundert währenden - Geschichte vermeintlich preisgegeben hat. Zunächst scheint kaum ein Zweifel daran zu bestehen: Goldgrub tut in seinen Fotografien alles, um die Auslöschung dessen, was Benjamin mit Aura gemeint haben mag, so eindrucksvoll wie möglich vorzuführen. Es ist, als wolle Goldgrub Benjamin mit Nachdruck in der Auffassung bestätigen, die Fotografie und vor allem sie sei in der Lage, jene Zertrümmerung der auratischen Reste der Moderne, die in den postmodernen Megacities ein durch den globalisierten Kapitalismus noch verstärktes, mittlerweile extrem gewordenes Ausmaß angenommen hat, zu dokumentieren. Die verlorene Aura der Stadt-Fotografie, so scheinen diese Arbeiten sagen zu wollen, ist allerdings weniger 'medial', weniger durch die fortgeschrittene Reproduktionstechnik der Fotografie bedingt als vielmehr durch die verlorene Aura der neuen Städte selbst. In den Fokus rückt ihr zusehends inhumaner, Menschen mehr und mehr auf Maschinen reduzierender Charakter.

Aber werfen wir einen Blick auf eine dieser Aufnahmen, deren deauratisierendem Effekt man sich kaum entziehen kann. Zum Beispiel jene Arbeit aus der Outdoors-Serie, in der die rechte Seite, fast bis zur Mitte, von einem senkrecht halbierten jungen Gesicht (dem eines Mädchens offenbar) ausgefüllt wird: einem so makellosen wie unheimlichen, von etwas Fernem erschreckten oder gebannten Plakatgesicht (Abb. 5). Wofür ein solches Gesicht geworben haben mag, lässt sich nicht mehr erschließen. Auf der linken Seite, parallel zur Front des Gesichts und von ihm durch einen Streifen Licht getrennt, ragt eine weitgehend fensterlose, mit kaum entzifferbaren Graffiti am oberen Rand 'verzierte' 
Hochhauskulisse auf, deren Schäbigkeit zu dem Gesichtsfragment in merkwürdigem Kontrast steht. Vergleicht man diese Aufnahme mit Arbeiten von Thomas Struth oder Gregory Crewdson, so fällt zweierlei auf: zum einen, dass das Prinzip gestaffelter Rahmungen, das bei Struth zur Steigerung des Aura-Effekts dient, hier nicht nur unterlaufen, sondern ausdrücklich aufgenommen und negiert wird. Das Gesicht des Mädchens wirkt, so gesehen, geradezu amputiert; die gestrichelte Rahmen-Andeutung, die das Foto im Foto umgibt - eine grobe Naht auf der Plakatwand -, macht das Fehlen der Rahmung besonders auffällig. Dieses Wegschneiden wird noch durch den Lichtschacht betont, diese leere weiße Fläche, die sich zwischen Gesicht und Hochhaus schiebt - mehr Abgrund als Himmel. Aber auch das Hochhaus kann kaum als Ersatz für die fortgefallene Rahmung gelesen werden; eher als Anspielung auf seine Ruinierung. Das anti-auratische Moment verstärkt sich durch den nicht eigentlich intermedialen, sondern interdisziplinären Charakter des Bildes. Hatten die trans- und intermedialen Inszenierungen bei Struth und Crewdson noch zur Verstärkung des Auratischen beigetragen, so trifft hier künstlerische auf kommerzielle Fotografie: Eine metareflexive Struktur, die einen sanften Schock auslöst - um diesen Begriff Benjamins aus dem Kunstwerk-Aufsatz zu verwenden -, weil der Kontext, mit dem das Foto das Foto umgibt, in der Sprengung jeglichen Kontexts besteht. Beschränkt man das halbierte Gesicht, dessen linkes Auge auf ein Geschehen über und hinter uns zielt und uns in seinen Schrecken mit hinein nimmt, nicht nur auf seinen Werbeauftrag, so deutet sich darüber hinaus auch eine transmediale Perspektive an. Und zwar, ähnlich wie bei Struth und Crewdson, in Richtung klassische Malerei. Man darf sich dann an das von einer Hand verdeckte, ebenfalls halbierte Gesicht des sog. 'Zeugen' auf Michelangelos Jüngstem Gericht in der Cappella sistina erinnern (Abb. 6). Diese Hock- oder Schwebefigur mit aufgerissenem Mund erschrickt möglicherweise mehr über das, was sich vor ihrem Auge abspielt - also im Bereich der Zuschauer, der Bildbetrachter - als über das Gerichtsgeschehen hinter ihr. Der Inszenierung 
der Aurazertrümmerung kämen so geradezu apokalyptische Dimensionen zu. Unentscheidbar bleibt dabei, ob das Erschrecken von der unmittelbaren Umgebung des Gesichts ausgelöst wird oder doch von all den anderen medialen Reproduktionen, in denen es sich spiegelt und die diese Stadt bebildern.

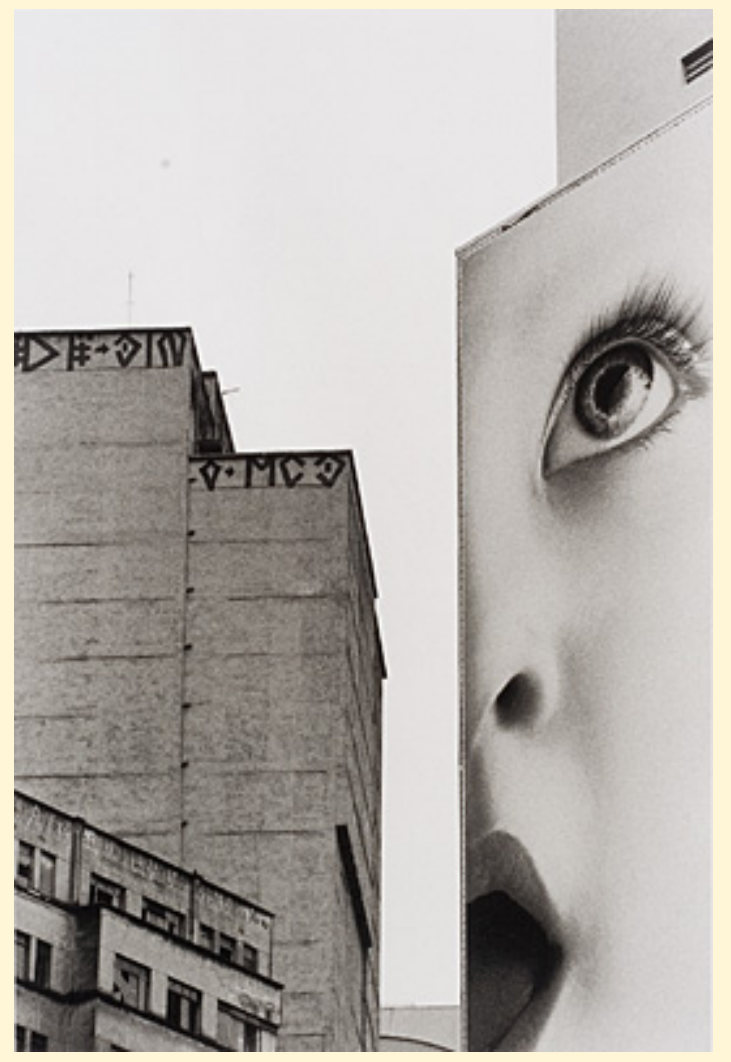

Abb. 5: Carlos Goldgrub, aus: Outdoors (São Paulo, 2003) 


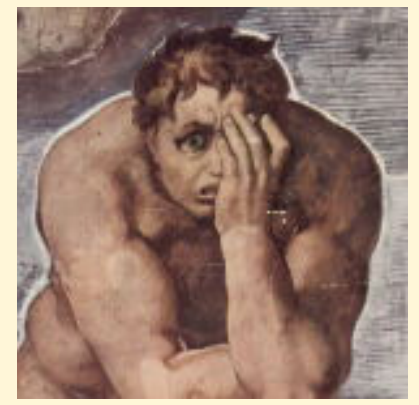

Abb. 6: Michelangelo, aus: Giudizio Universale, Cappella Sistina, Rom (1541)

Dennoch: Der antiauratische Impetus bleibt nicht die einzige Möglichkeit, Goldgrubs Fotografie zu interpretieren. Kehrt man nochmals zu Benjamins Photographie-Aufsatz zurück, so zeigt sich, dass der Aura-Begriff dort gerade im Zusammenhang mit Atget eingeführt wird - mit jenem Fotografen also, dessen Arbeit der Aura zum Verhängnis geworden sein soll. Benjamin schreibt an der entsprechenden Stelle, kurz, bevor er seine berühmte Aura'Definition' formuliert: „Er [Atget, U.J.B.] suchte das Verschollene und Verschlagene, und so wenden auch solche Bilder sich gegen den exotischen, prunkenden, romantischen Klang der Stadtnamen; sie saugen die Aura aus der Wirklichkeit wie Wasser aus einem sinkenden Schiff" (378; Herv. U.J.B.). Schon die wenige Zeilen zuvor hervorgehobene „Befreiung des Objekts von der Aura“, die er als das „Verdienst der jüngsten Photographenschule“ bezeichnet, lässt aufhorchen (ebd. 378). Wäre die Aura möglicherweise gar nicht jenes Menetekel der Moderne, als das sie in weiten Teilen des Textes erscheint? Die Metaphorik vom Schiffsuntergang führt ebenfalls in Paradoxien. Zum einen: Fotos, die die Aura aus der Wirklichkeit 'gesaugt' haben, haben diese dann offenbar nicht nur vernichtet, sondern auch adaptiert und sich einverleibt. Zum zweiten: Wenn Aura mit Wasser im sinkenden Schiff verglichen wird, so mit eben jenem Element, das gerade nicht die heile Welt jenseits des Verfalls darstellt, sondern selbst zum Verfall beiträgt, selbst Verfall ist. Das heißt nichts Anderes, als dass Spuren der 
Aura sich noch dort finden, wo sie bedroht und geschwächt wird - ja, dass selbst die Zerstörung der Aura noch 'Aura' haben kann. Indem Goldgrub die Deauratisierung in seinen Arbeiten bis zum Äußersten treibt, beschwört er - ex negativo, als Desiderat gerade das herauf, dessen Existenz er Metropolen wie Sao Paulo so vehement bestreitet: die Aura. Keine Aura mit Gebirgszügen und Zweigen freilich, sondern eine Aura der Entfremdung und der Einsamkeit, eine Anti- und Para-Aura, die die Perspektive der Differenz, die der Rede von der Aura immer schon inhärent ist, verabsolutiert.

\section{V}

Blickt man auf die verschiedenen, in unserem Experiment unterstellten 'Übertragungen' von Benjamins Kleiner Geschichte der Photographie zurück, so zeigt sich, dass Struth und Crewdson Benjamins Aura-Konzept zwar tendenziell folgen und seine Implikationen übernehmen, dass sie es aber gerade in jenem Medium zu erzeugen versuchen, das sich bei Benjamin auf Grund seiner genuinen Nähe zu Reproduktion und Kopie - ähnlich wie der Film - allem Auratischen widersetzt. Insofern ist ihre Nähe zu Benjamin trügerisch: Einerseits partizipieren sie wie selbstverständlich an seinem Diskurs, akzentuieren sie in ihren 'Übertragungen' das, was man Benjamins 'Thesen' nennen könnte und was bis heute von seinen kunsttheoretischen Texten zur 'Aura' kolportiert wird: das Kultische, das Mystische, das WerthaftBedeutsame. Andererseits scheint es ihnen, der Legitimierung ihres medialen Transfers wegen, auf jene weniger auffälligen Stellen anzukommen, an denen Benjamin „Technik und Magie“ miteinander in Verbindung bringt oder der Photographie in ihren „frühen Bildern“ die Möglichkeit des Auratischen einräumt (373). Heißt es nicht einmal sogar von den Lichtbildern, „die exakteste Technik“ könne „ihren Hervorbringungen einen magischen Wert geben, wie für uns ihn ein gemaltes Bild nie mehr besitzen kann“ 
(371)? Aber selbst der Fotograf, der die von Benjamin betonte deauratisierende Funktion der neueren Fotografie widerspruchslos zu akzeptieren scheint und dessen Übertragung man von daher fast 'wörtlich' nennen möchte, selbst Carlos Goldgrub kann - und will vielleicht auch - nicht vermeiden, dass sich in seinen Arbeiten auratische Effekte einstellen. Tragen bei Struth und Crewdson subtile transmediale Arrangements, gestaffelte Rahmungen, Miseen-abyme-Strukturen, semantische Verdichtungen und Momente der Fülle bzw. der Leere dazu bei, die Wirksamkeit der Auratisierung zu steigern, so sind es bei Goldgrub vor allem Überschreitungen innerhalb der fotografischen Genres - ein dekonstruktiver Umgang mit Rahmungen und Differenzen, der den Effekt einer Aura evoziert, deren Historizität irreversibel geworden ist. Eben darin zeichnen sich Parallelen ab: Mediale Aura ist zwar immer schon perspektivisch vermittelt und artifiziell hervorgebracht, wird aber jeweils als 'reales', 'authentisches' Ereignis inszeniert. Ihre künstlerische Herstellung zeichnet sich gerade dadurch aus, dass es ihr gelingt, die aller Medialität innewohnende Paradoxie von An- und Abwesenheit, Erscheinen und Verschwinden selbst zum Thema zu machen und aus dem Spiel mit historischer Differenz Energie zu gewinnen.

\section{Noten}

1. Uma versão ampliada deste artigo foi publicada em BEIL, U.J. Mediale Auren. Walter Benjamin und Fotografien von Thomas Struth, Gregory Crewdson und Carlos Goldgrub. In : BEIL, U.J., HERBERICHS, C., SANDL, M. (orgs. u. Mitarbeit von A. Bünder). Aura und Auratisierung. Mediologische Perspektiven im Anschluss an Walter Benjamin. Zurique: Chronos, 2014. p. 413-444. Col. Medienwandel - Medienwechsel - Medienwissen, Vol. 27.

Cad. Trad., Florianópolis, $n^{0}$ especial, p. 50-81, jul./dez. 2014 
2. Walter Benjamins zuerst 1931 in Die literarische Welt erschienener Essay Kleine Geschichte der Photographie, aus dem diese Passage entnommen ist, wird im Text unter Angabe der Seitenzahl zitiert nach: Walter Benjamin, Kleine Geschichte der Photographie, in: Gesammelte Schriften. Hg. von Rolf Tiedemann und Hermann Schweppenhäuser. Bd. II, 1 (werkausgabe Band 4), Frankfurt a. M. 1980 ff., S. 368-385, hier S. 378.

3. Karl Kraus / Otto Stoessl: Briefwechsel. 1902-1925, Wien 1996, S. 23. Der Satz wird von Benjamin mehrfach zitiert.

4. Zu den intertextuellen Spuren vor allem: Marleen Stoessel: Aura - das vergessene Menschliche. München/Wien 1983, S. 57 f.; Mika Elo: Die Wiederkehr der Aura, in: Christian Schulte (Hg.): Walter Benjamins Medientheorie. Konstanz 2005, S. 117-135, hier S. 121f.; Wolfgang Bock: Vom Blickwispern der Dinge. Sprache, Erinnerung und Ästhetik bei Walter Benjamin. Vorlesungen in Rio de Janeiro 2007. Würzburg 2010, S. 126 f. (und passim); sowie Josef Fürnkäs: Aura, in: Michael Opitz, Erdmut Wizisla (Hg.): Benjamins Begriffe. Bd. 1, Frankfurt/M. 2000, S. 95-146. Den Hinweis auf Riegl verdanke ich Hans-Georg von Arburg.

5. Walter Benjamin, Das Kunstwerk im Zeitalter seiner technischen Reproduzierbarkeit und weitere Dokumente. Kommentar von Detlev Schöttker. Frankfurt/M. 2007 (Suhrkamp Studienbibliothek 1), S. 16. Hier wird aus dem „Sommermittag“ ein „Sommernachmittag“, aus dem „Betrachter“ ein "Ruhende[r]“.

6. Vgl. Gershom Scholem: Die jüdische Mystik in ihren Hauptströmungen, Frankfurt/M. 1957, S. 311: „Jeder Mensch trägt die geheimen Spuren der Wanderung seiner Seele in den Lineamenten seiner Stirn und seiner Hand mit sich, sowie in der Aura, die von seinem Körper ausstrahlt. “ Zum metaphorischästhetischen Umgang Benjamins mit Motiven der kabbalistischen Tradition Andreas B. Kilcher: Der Sprachmythos der Kabbala und die ästhetische Moderne, in: Poetica 25 (1993), S. 237-261; Britta Leifeld: Das Denkbild bei Walter Benjamin. Die unsagbare Moderne als denkbares Bild, Frankfurt/M. u. a. 2000, S. 215-240; Sigrid Weigel: Walter Benjamin. Die Kreatur, das Heilige, die Bilder, Frankfurt/M. 2008; Ulrich Johannes Beil: Alterität, Aura, Präsenz. Mediale Konstellationen bei Hofmannsthal, Musil und Benjamin, in: ders., Michael Gamper, Karl Wagner (Hg.), Medien, Technik, Wissenschaft. Wissensübertragung bei Robert Musil und in seiner Zeit, Zürich 2011, S. 95-118. 
7. Für einen Überblick nach wie vor grundlegend: Fürnkäs, Aura, (Anm. 3); vgl. seitdem auch Elo, Die Wiederkehr der Aura (Anm. 3); Karl Clausberg, Elize Bisanz, Cornelius Weiller (Hg.): Ausdruck - Ausstrahlung - Aura. Synästhesien der Beseelung im Medienzeitalter, Bad Honnef 2007, bes. S. 29-39.

8. Anders Dieter Mersch: Ereignis und Aura. Untersuchungen zu einer Ästhetik des Performativen, Frankfurt/M. 2002, S. 109, der den Gegensatz zwischen einer 'unverfügbaren' und einer 'medial' vermittelten Aura betont.

9. Zu den Quellen Rolf H. Krauss: Walter Benjamin und der neue Blick auf die Photographie, Ostfildern 1998, S. 14-20.

10. Vgl. Bernd Stiegler: Theoriegeschichte der Photographie, München ${ }^{22} 210$, S. $258 f$.

11. In seinem Arbeitsjournal schreibt Brecht: „alles mystik, bei einer haltung gegen mystik. [...] Es ist ziemlich grauenhaft“; Bertolt Brecht, Arbeitsjournal, hg. von Werner Hecht, 2 Bde., Frankfurt/M. 1974, Bd. 1, S. 14. Antoine Hennion und Bruno Latour überschreiben einen Beitrag zum Kunstwerk-Aufsatz "How to make mistakes on so many things at once - and become famous for it", in: Hans-Ulrich Gumbrecht, Michael Marrinan (Hg.): Mapping Benjamin. The Work of Art in the Digital Age, Stanford 2003, S. 91-97.

12. Thomas Struth: Fotografien 1978-2010. Hg. von Anette Kruszynski, Tobia Bezzola, James Lingwood. Mit Texten von Armin Zweite u.a. München 2010, S. 198.

13. Struth, Fotografien 1978-2010 (Anm. 11), S. 131.

14. Abdruck z.B. in Walter Liedtke: Vermeer. The Complete Paintings. Antwerpen 2008, S. 101-103.

15. Walter Benjamin, Das Kunstwerk im Zeitalter seiner technischen Reproduzierbarkeit (Anm. 4), S. 23.

16. Stephan Berg: Die dunkle Seite des amerikanischen Traums / The Dark Side of the American Dream, in: Gregory Crewdson 1985-2005. Hg. von 
Stephan Berg. Mit Texten von Stephan Berg, Martin Hochleitner, Katy Siegel [Ausstellungskatalog]. Ostfildern 2005, S. 10-21.

17. Berg, Die dunkle Seite (Anm. 15), S. 18.

18. Martin Hochleitner: Zur Ikonographie des Lichts im Werk von Gregory Crewdson / On the Iconography of Light in the Works of Gregory Crewdson, in: Gregory Crewdson 1985-2005. Hg. von Stephan Berg. Mit Texten von Stephan Berg, Martin Hochleitner, Katy Siegel [Ausstellungskatalog]. Ostfildern 2005, S. 150-159, hier S. 150.

19. Abgedruckt ohne Seitenangabe in: Gregory Crewdson 1985-2005. Hg. von Stephan Berg. Mit Texten von Stephan Berg, Martin Hochleitner, Katy Siegel [Ausstellungskatalog]. Ostfildern 2005.

20. Vgl. Hochleitner, Zur Ikonographie des Lichts (Anm. 17), S. 152.

21. Abgedruckt in: Georges Didi-Huberman: Fra Angelico. Unähnlichkeit und Figuration. Aus dem Französischen von Andreas Knop. München 1995, S. 206.

22. Fritz Franz Vogel: The Cindy Shermans: inszenierte Identitäten. Fotogeschichten von 1840 bis 2005. Köln-Weimar-Wien 2006. S. 468.

23. Repräsentative Beispiele der Outdoor-Arbeiten finden sich u.a. auf der Website von Carlos Goldgrub (www.carlosgoldgrub.com), sowie in dem von der Kuratorin Dirce Carrion herausgegebenen Prospekt zur Ausstellung: Carlos Coldgrub: São Paulo - Um Retrato Urbano. São Paulo 2003.

24. So Helmut Galle: Vom urbanisierten Subjekt zur Zerstörung des Urbanen, in: Ralph Buchenhorst, Miguel Vedda (Hg.): Urbane Beobachtungen. Walter Benjamin und die neuen Städte. Übersetzt von Martin Schwietzke. Bielefeld 2010, S. 161-174, hier S. 169f; Mario de Andrade: Paulicéia desvairada, in: ders.: Poesias completas. Hg. von Diléa Zanotto Manfio. Belo Horizonte-São Paulo 1987, S. 55-115 (Orig.: 1922).

25. Carrion, Carlos Goldgrub (Anm. 22), S. 3. 
26. Márcio Seligmann-Silva: São Paulo. Zwei Fotografen, die eine unbewusste Optik der Stadt offenbaren, in: Ralph Buchenhorst, Miguel Vedda (Hg,) Walter Benjamin und die neuen Städte (Anm. 23), S. 187-207, hier S. 203. Ebd., S. 204. „Atgets Pariser Photos sind die Vorläufer der surrealistischen Photographie“: Benjamin, Kleine Geschichte der Photographie (Anm. 1), S. 378. Carrion, Carlos Goldgrub (Anm. 22), S. 2. Klaus Heinrich: Floß der Medusa, in: ders.: Floß der Medusa. Drei Studien zur Faszinationsgeschichte mit mehreren Beilagen und einem Anhang. Basel-Frankfurt a.M. 1995, S. 9-74, hier S. 38.

\section{References}

Andrade, Mario de. Poesias completas. Hg. von Diléa Zanotto Manfio. Belo Horizonte: Itatiaia, 1987.

Beil, Ulrich Johannes. „Alterität, Aura, Präsenz: Mediale Konstellationen bei Hofmannsthal, Musil und Benjamin“. In ___ Gamper, Michael; Wagner, Karl (Hg.). S. 95-118.Medien, Technik, Wissenschaft: Wissensübertragung bei Robert Musil und in seiner Zeit. Zürich: Chronos, 2011.

Benjamin, Walter. „Kleine Geschichte der Photographie“. S. 368-385. Bd. II, 1 (werkausgabe Band 4). In . Gesammelte Schriften. Herausgeber Rolf Tiedemann und Hermann Schweppenhäuser. Frankfurt am Main, 1980.

. Das Kunstwerk im Zeitalter seiner technischen Reproduzierbarkeit und weitere Dokumente. Kommentar von Detlev Schöttker. Frankfurt am Main: Suhrkamp Studienbibliothek 1, 2007.

Berg, Stephan (Hg.). Gregory Crewdson 1985-2005. Mit Texten von Stephan Berg, Martin Hochleitner, Katy Siegel [Ausstellungskatalog]. Ostfildern: Hatje Cantz Verlag, 2005.

(Hg.). „Die dunkle Seite des amerikanischen Traums / The Dark Side of the American Dream“. S. 10-21. In . Gregory Crewdson 1985-2005. Mit 
Texten von Stephan Berg, Martin Hochleitner, Katy Siegel [Ausstellungskatalog]. Ostfildern: Hatje Cantz Verlag, 2005. S. 10-21.

Bock, Wolfgang. Vom Blickwispern der Dinge: Sprache, Erinnerung und Ästhetik bei Walter Benjamin. Vorlesungen in Rio de Janeiro 2007. Würzburg: Königshausen \& Neumann 2010.

Brecht, Bertolt: Arbeitsjournal. 2 Bde. Hg. von Werner Hecht. Frankfurt am Main: Suhrkamp, 1974.

Carrion, Dirce. Carlos Coldgrub: São Paulo - Um Retrato Urbano [Ausstellungsprospekt]. São Paulo: [o.N.], 2003.

Clausberg, Karl; Bisanz, Elize; Weiller, Cornelius (Hg.): Ausdruck Ausstrahlung - Aura: Synästhesien der Beseelung im Medienzeitalter. Bad Honnef: Hippocampus, 2007.

Didi-Huberman, Georges. Fra Angelico: Unähnlichkeit und Figuration. Aus dem Französischen von Andreas Knop. München: Wilhelm Fink, 1995.

Elo, Mika. „Die Wiederkehr der Aura“. S. 117-135. In Schulte, Christian (Hg.). Walter Benjamins Medientheorie. Konstanz: UVK, 2005.

Fürnkäs, Josef. „Aura“. In Opitz, Michael; Wizisla, Erdmut (Hg.). S. 95-146. Bd. 1. Benjamins Begriffe. Frankfurt am Main: Suhrkamp, 2000.

Galle, Helmut. „Vom urbanisierten Subjekt zur Zerstörung des Urbanen“. S. 161-174. In Buchenhorst, Ralph; Vedda, Miguel (Hg.). Urbane Beobachtungen: Walter Benjamin und die neuen Städte. Übersetzt von Martin Schwietzke. Bielefeld: Transcript, 2010.

Gumbrecht, Hans-Ulrich; Marrinan, Michael (Hg.). Mapping Benjamin: The Work of Art in the Digital Age. Stanford: Stanford University Press, 2003.

Heinrich, Klaus. Floß der Medusa: Drei Studien zur Faszinationsgeschichte mit mehreren Beilagen und einem Anhang. Frankfurt am Main: Stroemfeld, 1995. 
Hochleitner, Martin. „Zur Ikonographie des Lichts im Werk von Gregory Crewdson / On the Iconography of Light in the Works of Gregory Crewdson“. S. 150-159. In Berg, Stephan (Hg.). Gregory Crewdson 1985-2005. Mit Texten von Stephan Berg, Martin Hochleitner, Katy Siegel [Ausstellungskatalog]. Ostfildern: Hatje Cantz, 2005.

Kampff Lages, Susana. Walter Benjamin: Tradução e Melancolia. São Paulo: Edusp, 2002.

Kilcher, Andreas B. „Der Sprachmythos der Kabbala und die ästhetische Moderne“. Bd. 25 (1993), S. 237-261. Poetica, Leipzig.

Kraus, Karl; Stoessl, Otto. Briefwechsel 1902-1925. Wien: Deuticke, 1996.

Krauss, Rolf H. Walter Benjamin und der neue Blick auf die Photographie. Ostfildern: Hatje Cantz, 1998.

Leifeld, Britta. Das Denkbild bei Walter Benjamin: Die unsagbare Moderne als denkbares Bild. Frankfurt am Main: Peter Lang, 2000.

Liedtke, Walter. Vermeer: The Complete Paintings. Antwerpen: Luidon, 2008.

Mersch, Dieter. Ereignis und Aura: Untersuchungen zu einer Ästhetik des Performativen. Frankfurt am Main: Suhrkamp, 2002.

Scholem, Gershom. Die jüdische Mystik in ihren Hauptströmungen. Frankfurt am Main: Suhrkamp, 1957.

Seligmann-Silva, Márcio. „São Paulo: Zwei Fotografen, die eine unbewusste Optik der Stadt offenbaren“. S. 187-207. In Buchenhorst, Ralph; Vedda, Miguel (Hg,). Urbane Beobachtungen: Walter Benjamin und die neuen Städte. Übersetzt von Martin Schwietzke. Bielefeld: Transcript, 2010.

Stiegler, Bernd. Theoriegeschichte der Photographie. München: Wilhelm Fink, 2010 . 
Stoessel, Marleen: Aura: das vergessene Menschliche. München: Hanser, 1983.

Struth, Thomas. Fotografien 1978-2010. Hg. von Anette Kruszynski, Tobia Bezzola, James Lingwood. Mit Texten von Armin Zweite. München: Schirmer/ Mosel, 2010.

Vogel, Fritz Franz. The Cindy Shermans: inszenierte Identitäten. Fotogeschichten von 1840 bis 2005. Köln: Böhlau, 2006.

Weigel, Sigrid. Walter Benjamin: Die Kreatur, das Heilige, die Bilder. Frankfurt am Main: Fischer, 2008.

Recebido em: 12/02/2014

Aceito em: 23/05/2014 